\title{
Métodos de Registro de Dimensión Vertical Oclusal en Pacientes Dentados: Revisión Sistemática
}

\author{
Registration Methods of Occlusal Vertical Dimension \\ in Dentate Patients: Systematic Review
}

\author{
Olivia Alatorre Castorena1; José Velasco Neri²,3; Verónica Manteca López²; \\ Daniel Llamas Haro ${ }^{2}$ \& Andréa Dolores Correia Miranda Valdivia ${ }^{4}$
}

\begin{abstract}
ALATORRE, C. O.; VELASCO, N. J.; MANTECA, L. V.; LLAMAS, H. D. \& VALDIVIA, A. D. C. M. Métodos de registro de dimensión vertical oclusal en pacientes dentados: Revisión sistemática. Int. J. Odontostomat., 15(2):397-402, 2021.

RESUMEN: La Dimensión Vertical Oclusal, es la distancia entre un punto anatómico fijo en el maxilar y otro sobre la parte móvil de la mandíbula cuando los órganos dentarios están en contacto oclusal. La obtención de este registro determina el espacio vertical necesario para la rehabilitación de los pacientes. En la actualidad existen distintos métodos para el registro de la Dimensión Vertical Oclusal, en este estudio se analizaron: Métodos fisiológicos y métodos mecánicos. El objetivo fue realizar una revisión sistemática, sobre los métodos de registro, para determinar la Dimensión Vertical Oclusal en pacientes dentados. Se recopiló información científica desde Mayo 2019 hasta Julio 2020 en diferentes bases de datos electrónicas, encontrando un total de 13948 artículos, después de eliminar artículos repetidos, por título, por resumen y por metodología PICO se seleccionaron un total de 24 artículos para su análisis y clasificación. Más de un autor menciona que no existe un método unirversalmente aceptado o exacto para determinar la Dimensión Vertical Oclusal. Se sugiere la combinación de varios métodos de registro de Dimensión Vertical Oclusal, se debe de considerar la manera más adecuada para lograr un registro más acertado.
\end{abstract} vertical.

PALABRAS CLAVE: Dimensión vertical de oclusión; puntos de referencia anatómicos, oclusón dental, dimensión

\section{INTRODUCCIÓN}

En Prostodoncia existen dos tipos de Dimensión Vertical (DV), la Dimensión Vertical Oclusal (DVO) y la Dimensión Vertical de Reposo (DVR). La DVO, es la distancia entre un punto anatómico fijo en el maxilar (punta de la nariz) y otro sobre la parte móvil de la mandíbula (mentón) cuando los órganos dentarios están en contacto oclusal. La DVR se define como la posición postural de la mandíbula, cuando un individuo esta en una posición confortable, vertical, estando los músculos relacionados en un estado de actividad contractural mínima (Ferro, 2017). La obtención de estos registros deben de ser lo mas confiables posibles, ya que determina el espacio vertical necesario para la rehabilitación de los pacientes dentados, par- cialmente dentados y totalmente edéntulos (Hurst, 1962; Aboul-Ela \& Abdel Razek, 1977).

En la actualidad existen distintos métodos para el registro de la DVO, en este estudio se analizaron dos métodos: los métodos fisiológicos y los métodos mecánicos; los primeros se pueden dividir en tres tipos: deglución, fonética y posición fisiológica de reposo. El segundo grupo se compone de 4 tipos: el primero es el de Tercios faciales de Willis, el segundo es el Craneómetro de Knebelman, el tercero el fotográfico, y el cuarto Craneómetro de Morata (VD-Meter) (Willis, 1935; McGee, 1947; Silverman, 1965; Swerdlow, 1965; Turner, 1969; Weinberg, 1982;

${ }^{1}$ Cirujano Dentista, Residente en Posgrado Prostodoncia, Universidad Autónoma de Guadalajara. Guadalajara, Jalisco, México.

${ }^{2}$ Cirujano Dentista, Especialista en Rehabilitación Oral, Profesor en Posgrado Prostodoncia, Universidad Autónoma de Guadalajara. Guadalajara, Jalisco, México.

${ }^{3}$ Doctor en Ciencias, Profesor Investigador en el Departamento de Clínicas Odontológicas Integrales del Centro Universitario de Ciencias de la Salud, Universidad de Guadalajara. Guadalajara,Jalisco, México.

${ }^{4}$ Doctora en Clinica Odontológica Integrada, Profesora Investigadora en el Departamento de Odontologia Especializada, Posgrado Prostodoncia, Universidad Autónoma de Guadalajara. Guadalajara, Jalisco, México. 
Tabla I. Método de Búsqueda PICO.

\begin{tabular}{ll}
\hline Método de búsqueda sistematizada \\
\hline Pregunta dirigida & ¿Cuál es la descripción de los métodos de registro de Dimensión Vertical de Oclusión (DVO) \\
PICO? (Patient or Population, Intervention, Control or Comparison and Outcome) \\
$\begin{array}{ll}\text { Participantes } & \text { Pacientes dentados } \\
\text { Intervención } & \text { Métodos de registro de DVO } \\
\text { Comparación } & \text { Diferentes métodos de registro de DVO } \\
\text { Resultados } & \text { Descripción de los métodos de registro de DVO } \\
\text { Bases de datos } & \text { SciELO, PUBMED, EBSCO, Dialnet, BVS, Book SC, MeSH, ClinicalTrials.gov, Trip Medical } \\
\text { Otros recursos } & \text { Obtenidos a través de otros medios. }\end{array}$ \\
\hline
\end{tabular}

Knebelman, 1988; Silverman, 2001; Tavano et al., 2012; Morata et al., 2020).

El objetivo de este manuscrito fue realizar una revisión sistemática de literatura sobre los métodos de registro fisiológicos y mecánicos para determinar la Dimensión Vertical de Oclusión en pacientes dentados.

\section{MATERIAL Y MÉTODO}

Se realizó una revisión sistemática utilizando el método de la Universidad de Oxford "Patient or Population, Intervention, Control or Comparision and Outcome" (PICO) (Tabla I). Fueron realizadas las bús-

Tabla II. Tipos de registro de DVO.

\begin{tabular}{|c|c|c|}
\hline $\begin{array}{l}\text { Tipo de } \\
\text { registro }\end{array}$ & Método de registro de DVO & Autor \\
\hline \multirow{6}{*}{$\begin{array}{l}\text { Subjetivo } \\
\text { (Fisiológico) }\end{array}$} & Método de deglución & (McGee, 1947) \\
\hline & & (Silverman, 1965, 2001) \\
\hline & Método fonético & (McGee, 1947) \\
\hline & & (Silverman, 1965, 2001) \\
\hline & & (Weinberg, 1982) \\
\hline & Reposo menos $3 \mathrm{~mm}$ & (Burnett, 2000) \\
\hline \multirow{13}{*}{$\begin{array}{l}\text { Objetivo } \\
\text { (Mecánico) }\end{array}$} & Método fotográfico & $\begin{array}{l}\text { (Baba et al., 2000) } \\
\text { (Vinnakota et al., 2016) } \\
\text { (Gomes et al., 2008) }\end{array}$ \\
\hline & & (Bajunaid et al., 2017) \\
\hline & Antropométrico & $\begin{array}{l}\text { (Nagpal et al., 2014) } \\
\text { (Boyan, 1970) }\end{array}$ \\
\hline & & $\begin{array}{l}\text { (Majeed et al., 2015, 2018) } \\
\text { (Ladda et al., 2014) } \\
\text { (Deli et al., 2003) }\end{array}$ \\
\hline & & (Tavano et al., 2012) \\
\hline & Cefalométrico & (Orthlieb et al., 2000) \\
\hline & ectarumedutco & $\begin{array}{l}\text { (Yamashita et al., 2015) } \\
\text { (McGee, 1947) }\end{array}$ \\
\hline & & (Legan \& Burstone, 1980) \\
\hline & Método de Willis & (Geerts et al., 2004) \\
\hline & Craneómetro de & (Chou et al., 1994) \\
\hline & Knebelman & (Knebelman, 1980) \\
\hline & Craneómetro de Morata & (Morata et al., 2020) \\
\hline & Pre-extracción & (Aboul-Ela \& Razek, 1977) \\
\hline
\end{tabular}

quedas electrónicas de artículos relacionados a los diferentes métodos de registro de DVO utilizando las palabras clave: Occlusal vertical dimensión, methods occlusal vertical dimension, technique occlusal vertical dimensión, vertical dimensión of occlusion, dentate patient, toothed patient en 10 bases de datos: SciELO, PUBMED, Google Académico, Chochrane, BVS, Book SC, MeSH, ClinicalTrials.gov, Trip Medical Datebase, Trip Medical Datebase PICO.

\section{RESULTADOS}

Los resultados de la revisión sistemática realizados se podrán observan en un flujograma presentado en la Figura 1. Para la selección de artículos por resumen se determinaron los siguientes criterios de inclusión: artículos disponibles, artículos en inglés, estudios publicados de 1953 a julio 2019, estudios que presentaran una descripción clara del método de registro de DVO, participación de pacientes dentados. Los criterios de exclusión fueron: artículos con su contenido no disponible, artículos que no estén en el idioma inglés, métodos de DVO probados en pacientes edéntulos.

Se recopiló información científica desde Mayo 2019 hasta Julio 2020 en diferentes bases de datos electrónicas, encontrando un total de 13948 artículos, después de eliminar artículos repetidos, por título, por resumen y por metodología PICO se seleccionaron un total de 24 artículos para su análisis y clasificación. Los árticulos examinados en esta revisión sistemática fueron divididos según el tipo de registro, según el método de registro de DVO y por autor. Dicha clasificación se puede observar en la Tabla II. 
ALATORRE, C. O.; VELASCO, N. J.; MANTECA, L. V.; LLAMAS, H. D. \& VALDIVIA, A. D. C. M. Métodos de registro de dimensión vertical oclusal en pacientes dentados: Revisión sistemática. Int. J. Odontostomat., 15(2):397-402, 2021.

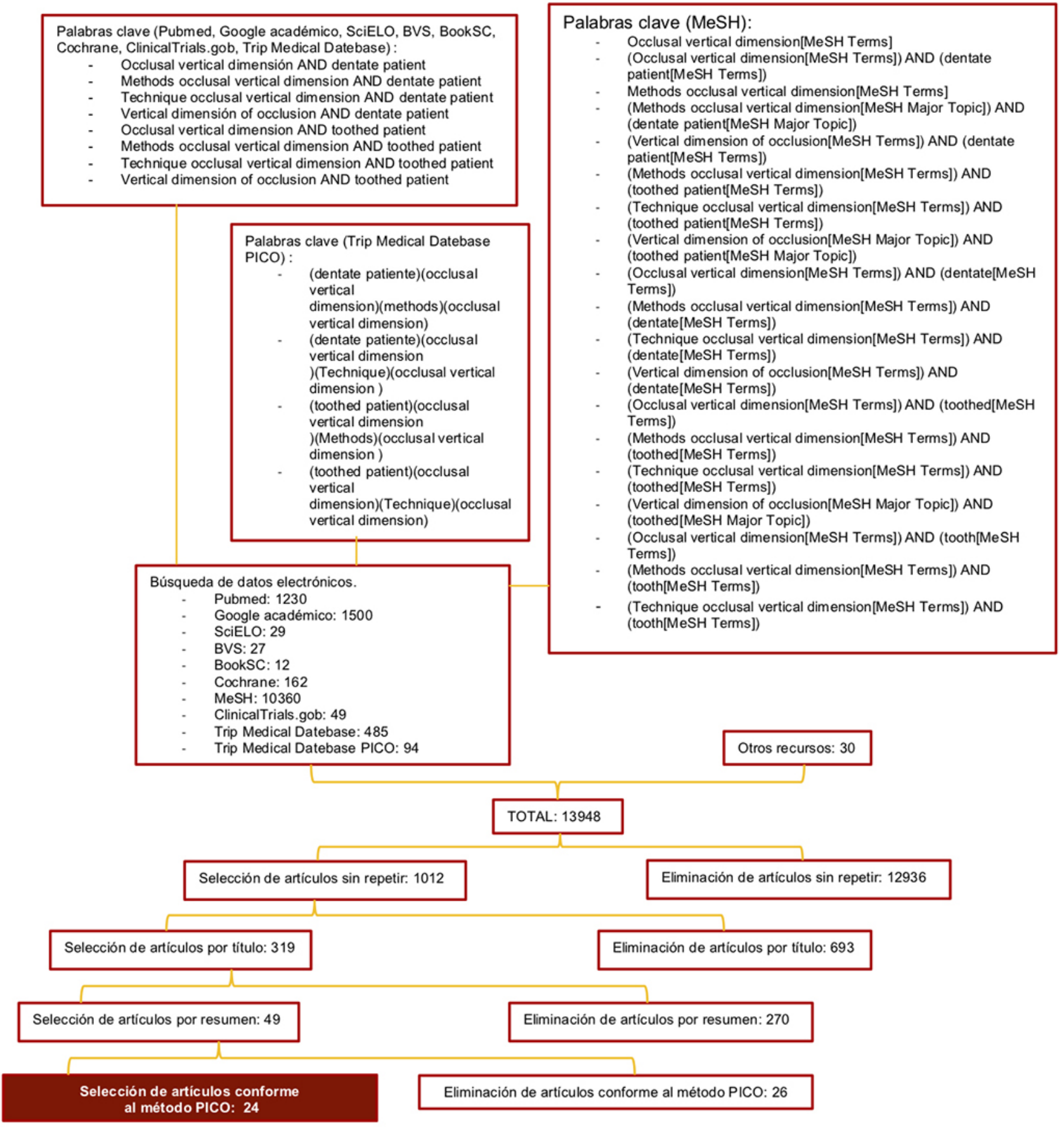

Fig. 1. Flujograma de Resultados

\section{DISCUSIÓN}

En esta revisión sistemática, la definición en la que todos los autores coinciden de la DVO es la distancia entre dos puntos anatómicos normalmente uno en la punta de la nariz y el otro en el mentón cuando está en máxima intercuspidación, no menciona si esta máxima intercuspidación debería coincidir con relación céntrica (Ferro); considerando que un espacio interoclusal adecuado sea entre 1 a $3 \mathrm{~mm}$ (Baba et al., 
2000). Alhajj et al. (2017) mencionan que para evitar el fracaso de las restauraciones en prostodoncia es importante determinar correctamente la DVO. Bajunaid et al. (2017) clasificaron el registro de la DVO, en métodos subjetivos y métodos objetivos.

Los métodos subjetivos son los que incluyen evaluaciones de la posición de reposo fisiológico, deglución, fonética y estética; mientras que los métodos objetivos incluyen evaluaciones de mediciones faciales, registros previos a la extracción, cefalogramas y electromiogramas (Bajunaid et al.). Los métodos de preextracción se basan en la suposición que el paciente presenta; estos métodos solo se consideran adecuados si las mediciones de la DVO se realizan en pacientes dentados con una oclusión aceptable (Alhajj et al.).

Weingberg (1982) menciona que la DVR se atribuye a tres mecanismos posibles: tonicidad postural de los músculos, reflejos miostáticos y elasticidad o una combinación de las tres (Yamashita et al., 2015). Burnett (2000) describe la posición fisiológica de reposo como un punto de referencia comúnmente utilizado en la determinación de DVO, menciona que se considera como un rango, afectado por una serie de factores, en lugar de uno absoluto tal como lo menciona Tallgren (1957) y Wessberg et al. (1983) (Tallgren, 1957; Wessberg et al.; Burnett). En un estudio de Thomson y Kendrick demostraron cambios significativos de la DV en todos sus pacientes (Thompson \& Kendrick, 1964). Atwood (1966), Tallgreen (1972) y Bajunaid et al. mencionan que la posición fisiológica de reposo no es consistente, por lo que no es una posición de referencia confiable para determinar la DVO (Atwood; Tallgren, 2003; Bajunaid et al.). Mientras que en un estudio electromiográfico Garnick demuestra que la variación de una posición de descanso es de $1.5 \mathrm{~mm}$ (Garnick, 1962). Darling demostró en un estudio que la postura habitual de la cabeza o la mala posición de la cabeza pueden causar una alteración de la DVR (Darling et al., 1984).

McGee describió y probó en pacientes dentados distintos métodos de DVO. Describió el método fonético como ciertos sonidos de letras y palabras realizados por el paciente que se utilizan para determinar el espacio entre los dientes y el espacio interoclusal. Otro método es permitir que el paciente determine la posición más cómoda. Así mismo, el método de deglución, lo describe para producir el límite superior del espacio libre. Los métodos cefalométricos los describe como un método científico para registrar la DVO pre-extracción (McGee).
El método fonético para medir la DVO es un método de estimulación fisiológica de la actividad muscular durante la producción de sonidos del habla, que mide la dimensión vertical por medio del espacio de habla más cercano. El espacio libre establece la dimensión vertical cuando los músculos involucrados están en reposo completo y la mandíbula está en posición de reposo. Este espacio es también el medio de demostrar que no se debe aumentar la DVO (Silverman, 1965, 2001). Chou et al. (1994), confirman que los resultados son consistentes en el método fonético en pacientes clase I.

La DVO es el resultado de un equilibrio músculo esquelético durante el crecimiento, así lo describe Orthlieb et al. (2000), en un estudio dónde utiliza mediciones cefalométricas para probar la significancia estadística de las correlaciones entre la forma de la mandíbula y la DVO. Tavano et al. realizaron un análisis cefalométrico lateral llamado Seraidarian-Tavano, que se verificó a través de ángulos faciales (ángulos superiores y medios) que cuando se correlacionan, determinan el tercio inferior de la cara; coincidiendo así con Willis (1935) quien propuso una metodología que intentaba establecer la armonía entre los tercios superior, medio e inferior de la cara, mediante parámetros culturales.

El estudio de Tavano et al. intentó desarrollar una metodología que pudiera ser aplicable a cualquier individuo adulto, independientemente de su género o etnia, ya que afirma que los puntos de referencia encontrados en el tejido blando son inestables y no se pueden definir, es por eso que el uso de referencias óseas aumenta la precisión de estas mediciones. Mientras McGee y Pound (1966) demostraron en sus estudios que las mediciones en los tejidos blandos demostraban una alta predictibilidad (Wills Francis M, 1935; McGee; Pound; Tavano et al.). De igual forma Yamashita et al. en un estudio trataron de derivar una nueva fórmula para DVO utilizando el análisis cefalométrico, pero debido a la irregularidad de la raza de la población de la muestra se debe adicionar al estudio diferentes etnias y no solo radiografías laterales, también frontales (Yamashita et al.).

En lo que se refiere al método craneómetrico, algunos autores coinciden que no existe una cara verdaderamente simétrica, por lo que la asimetría "normal" no existe, por lo tanto, el método cefalométrico no es fiable debido a las variaciones individuales de una población (Thompson, 1943; McGee; Delic' et al., 2003). Así mismo Chou et al. 
menciona que las asimetrías de la cara hacen que las mediciones de los métodos craneométricos sean cuestionables (Chou et al.). Por el contrario Bajunaid et al. y Majeed et al. (2015) clasifican los registros craneofaciales como un método objetivo; y mencionan que existe una relación significativa entre la DVO y las distancias craneofaciales (Majeed et al., 2015; Bajunaid et al.).

Según Knebelman, Chou et al., Nagpal et al. (2014), Majeed et al. (2015), Bajunaid et al. y Morata et al. existen várias correlaciones craneofaciales con la DVO comprobadas por varios autores como la distancia ojo-oreja (Knebelman; Chou et al.; Nagpal et al.; Bajunaid et al.; Morata et al.), distancia de la nariz al mentón (Mish; Willis; Knebelman; Aldhaher HA; Nagpur), distancia interpupilar (Ladda et al., 2014); canto externo del ojo izquierdo a la comisura izquierda de la boca (Nagpal et al.); comisura labial derecha a comisura labial izquierda (McGee; Majeed et al., 2015); canto interno del ojo izquierdo a canto interno de ojo derecho multiplicado por dos (Majeed et al., 2015). Existe una variación considerable genotípica y fenotípica entre grupos étnicos y razas (Majeed et al. 2018), por lo tanto para utilizar las correlaciones craneofaciales es prudente analizarla y realizarla en una sola población.

Más de un autor menciona que no existe un método universalmente aceptado o exacto para determinar la DVO (Fayz \& Eslami, 1988; Bissasu, 1999); debido a eso al terminar el análisis de estos artículos podemos concluir que no existen comparaciones que determinen con exactitud la medida de la DVO real, esto debido a que los estudios se realizan en grupos de edad, étnicos y poblaciones distintas; y con diferentes puntos de referencia en los pacientes. El odontólogo debe de considerar las limitaciones y la capacidad de los métodos tanto subjetivos como objetivos. Así mismo, sugerimos la combinación de varios métodos de registro de DVO, se debe considerar la manera más adecuada para lograr un registro más acertado.

\section{AGRADECIMIENTOS}

La presente investigación fue realizada bajo la supervisión de docentes de la especialidad de Prostodoncia de la Universidad Autónoma de Guadalajara, a quienes agradecemos todo el apoyo brindado.
ALATORRE, C. O.; VELASCO, N. J.; MANTECA, L. V.; LLAMAS, H. D. \& VALDIVIA, A. D. C. M. Registration methods of occlusal vertical dimension in dentate patients: Systematic review. Int. J. Odontostomat., 15(2):397-402, 2021.

ABSTRACT: Occlusal Vertical Dimension is the distance between an anatomical point in the maxilla and another on the mobile part of the mandible when the teeth are in occlusal contact. Obtaining this record determines the vertical space necessary for the rehabilitation of patients. Nowadays there are different methods for the record of the Occlusal Vertical Dimension, in this study the following were analyzed: physiological methods and mechanical methods. The objective was to do a systematic review of the registration methods to determine the Occlusal Vertical Dimension in dentate patients. Scientific information was collected from May 2019 to July 2020 in different electronic databases, finding a total of 13,948 articles, after eliminating repeated articles, by title, by abstract and by PICO methodology, a total of 24 articles were selected for analysis and classification. More than one author mentions that there is no universally accepted or exact method for determining Occlusal Vertical Dimension. A combination of several methods of Occlusal Vertical Dimension registration is suggested; it should be considered the most appropriate way to achieve a more accurate registration.

KEY WORDS: vertical dimension of occlusion, anatomic landmarks, dental occlusion, vertical dimension.

\section{REFERENCIAS BIBLIOGRÁFICAS}

Aboul-Ela, L. M. \& Razek, M. K. Pre-extraction records of the occlusal plane and vertical dimension. J. Prosthet. Dent., 38(5):490-93, 1977.

Alhajj, M. N.; Khalifa, N.; Abduo, J.; Amran, A. \& Ismail I. A. Determination of occlusal vertical dimension for complete dentures patients: an updated review. J. Oral. Rehabil., 44(11):896-907, 2017.

Atwood, D. A. A critique of research of the rest position of the mandible. J. Prosthet. Dent., 16(5):848-54, 1966.

Baba, K.; Tsukiyama, Y. \& Clark, G. T. Reliability, validity, and utility of various occlusal measurement methods and techniques. $J$. Prosthet. Dent., 83(1):83-9, 2000.

Bajunaid, S. O.; Baras, B., Alhathlol, N. \& Ghamdi, A. Al. Evaluating the reliability of facial and hand measurements in determining the vertical dimension of occlusion. Int. J. Med. Pharm., 5(1):111, 2017.

Bissasu, M. Use of lingual frenum in determining the original vertical position of mandibular anterior teeth. J. Prosthet. Dent., 82(2):177-81, 1999.

Boyan, B. Determining vertical centric relation. J. Prosthet. Dent., 24(1):18-24, 1970.

Burnett, C. A. Clinical rest and closest speech positions in the determination of occlusal vertical dimension. J. Oral. Rehabil., 27(8):714-9, 2000. 
Chou, T.; Moore, D. J.; Young, L. \& Glaros, A. G. Removable Prosthodontics A diagnostic craniometric vertical dimension method for determining occlusal. J. Prosthet. Dent., 71(6):56874, 1994.

Darling, D. W.; Kraus, S. \& Glasheen-Wray, M. B. Relationship of head posture and the rest position of the mandible. J. Prosthet. Dent., 52(1):111-5, 1984.

Delic', Z.; Vukovojac, S.; Grzic, R.; Maricic, D.; Kovac, Z. \& Kovacevic', D. Evaluation of craniometric methods for determination of vertical dimension of occlusion--Part 2. Coll. Antropol., 27 Suppl. 1:191-4, 2003.

Fayz, F. \& Eslami, A. Determination of occlusal vertical dimension: A literature review. J. Prosthet. Dent., 59(3):321-3, 1988.

Ferro, K. J. (Ed.). The Glossary of Prosthodontic Terms. GPT-9. 9th ed. The Academy of Prosthodontics, Elsevier, The Journal of Prosthetic Dentistry, 117(5S), 2017.

Garnick, J. S. R. An electromyographic and clinical investigation. J. Prosthet. Dent., 12(5):895-911, 1962.

Geerts, G. A. V. M.; Stuhlinger, M. E. \& Nel, D. G. A comparison of the accuracy of two methods used by pre-doctoral students to measure vertical dimension. J. Prosthet. Dent., 91(1):59-66, 2004.

Gomes, V. L.; Gonçalves, L. C.; Correia, C. L.; Lucas, B. L. \& Carvalho, P. M. Vertical dimension of the face analyzed by digital photographs. Eur. J. Esthet. Dent., 3(4):362-70, 2008.

Hurst, W. W. Vertical dimension and its correlation with lip length and interocclusal distance. J. Am. Dent. Assoc., 64(4):496-504, 1962.

Knebelman, S. EE.UU. Patente $N^{\circ} 4,718,850$ Washington (D.C.), U.S. Patent and Trademark Office, 1988.

Ladda, R.; Kasat, V. O. \& Bhandari, A. J. A new technique to determine vertical dimension of occlusion from anthropometric measurement of interpupillary distance. J. Clin. Exp. Dent., 6(4):e395-9, 2014.

Legan, H. L. \& Burstone, C. J. Soft tissue cephalometric analysis for orthognathic surgery. J. Oral. Surg., 38(10):744-51, 1980.

Majeed, M. I.; Afzal, M. \& Kashif, M. Determination of occlusal vertical dimension in a section of Pakistani population using craniofacial measurements. J. Univ. Med. Dent. Coll., 6(1):1-5, 2015.

Majeed, M. I.; Haralur, S. B.; Khan, M. F.; Al Ahmari, M. A.; Al Shahrani, N. F. \& Shaik, S. An anthropometric study of craniofacial measurements and their correlation with vertical dimension of occlusion among Saudi Arabian subpopulations. Open Access Maced. J. Med. Sci., 6(4):680-6, 2018.

McGee, G. F. Use of facial measurements in determinig vertical dimension. J. Am. Dent. Assoc., 35(5):342-50, 1947.

Morata, C.; Pizarro, A.; Gonzalez, H. \& Frugone-Zambra, R. A craniometry-based predictive model to determine occlusal vertical dimension. J. Prosthet. Dent., 123(4):611-7, 2020.

Nagpal, A.; Parkash, H.; Bhargava, A. \& Chittaranjan, B. Reliability of different facial measurements for determination of vertical dimension of occlusion in edentulous using accepted facial dimensions recorded from dentulous subjects. J. Indian Prosthodont. Soc., 14(3):233-42, 2014.

Orthlieb, J. D.; Laurent, M. \& Laplanche, O. Cephalometric estimation of vertical dimension of occlusion. J. Oral. Rehabil., 27(9):8027, 2000.

Pound, E. The mandibular movements of speech and their seven related values. J. Prosthet. Dent., 16(5):835-43, 1966.

Silverman, M. M. Determination of vertical dimension by phonetics. J. Prosthet. Dent., 6(4):465-71, 1956.

Silverman, M. M. The speaking method in measure vertical dimension. 1952. J. Prosthet. Dent., 85(5):427-31, 2001.

Swerdlow, H. Vertical dimension literature review. J. Prosthet. Dent., 15:241-7, 1965.
Tallgren, A. Changes in adult face height due to aging, wear and loss of teeth and prosthetic treatment. Helsinki. Acta Odontologica Scandanavia, Supplement 24, 1957.

Tallgren, $A$. The continuing reduction of the residual alveolar ridges in complete denture wearers: A mixed-longitudinal study covering 25 years. J. Prosthet. Dent., 89(5):427-35, 2003.

Tavano, K. T. A.; Seraidarian, P. I.; De Oliveira, D. D. \& Jansen, W. C. Determination of vertical dimension of occlusion in dentate patients by cephalometric analysis - Pilot study. Gerodontology, 29(2):e297-305, 2012.

Thompson, J. L. \& Kendrick, G. S. Changes in the vertical dimensions of the human male skull during the third and fourth decades of life. Anat. Rec., 150(2):209-13, 1964.

Thompson, J. R. Asymmetry of the face. J. Am. Dent. Assoc., 30(23):1859-71, 1943.

Turner, L. C. The profile tracer: Method for obtaining accurate preextracion records. J. Prosthet. Dent., 21(4):364-70, 1969.

Vinnakota, D. N.; Kanneganti, K. C.; Pulagam, M. \& Keerthi, G. K. Determination of vertical dimension of occlusion using lateral profile photographs: A pilot study. J. Indian Prosthodont. Soc., 16(4):323-7, 2016.

Weinberg, L. A. Vertical dimension: A research and clinical analysis. J. Prosthet. Dent., 47(3):290-302, 1982.

Wessberg, G. A.; Epker, B. N. \& Elliott, A. C. Comparison of mandibular rest positions induced by phonetics, transcutaneous electrical stimulation, and masticatory electromyography. $J$. Prosthet. Dent., 49(1):100-5, 1983.

Willis, F. M. Features of the face involved in full denture prosthesis. Dent. Cosm., 77(9):851-4, 1935.

Yamashita, S.; Shimizu, M. \& Hidenori, K. A newly proposed method to predict optimum occlusal vertical dimension. J. Prosthodont., 24(4):287-90, 2015

Dirección para correspondencia:

Andréa Dolores Correia Miranda Valdivia DDS, MSc, PhD

Universidad Autónoma de Guadalajara

Profesora Facultad de Odontología

Especialidad en Prostodoncia.

Calle Escorza, \#526

Colonia Moderna. CP. 44150

Guadalajara

Jalisco

MÉXICO

Email: andrea.correia@edu.uag.mx 